\title{
Ultrafine Structure of Human Aortic Valve Calcific Deposits
}

\section{Felix Grases ${ }^{1 *}$, Otakar Sohnel ${ }^{2}$ and Marketa Zelenkova}

${ }^{1}$ Laboratory of Renal Lithiasis Research, University Institute of Health Sciences Research (IUNICS), University of Balearic Islands, Palma of Mallorca, Spain 2 University of J.E. Purkyně, Faculty of Environmental Studies, Ústí n.L., Czech Republic

${ }^{3}$ Synpo a.s., S.K. Neumanna, Pardubice, Czech Republic

\begin{abstract}
Objective: To elucidate the mechanism of formation of calcific deposits inside the human natural heart valve based on their ultra-fine structure on nanometer scale observed by atomic force microscope.

Methods: Cross-sections of an aortic valve calcification were observed by scanning electron microscope. Thin slices several micrometeres thick from the same deposit were observed by atomic force microscopy in the Peak Force Imagining mode providing topographic, adhesion and quantitative nanoscale elastic modulus maps of the surface.

Results: Most of the aortic valve calcification was composed of large blocks of mostly compact matter, porous on the microscopic scale. The mineral blocks consisted mainly of closely arranged elongated needle- and plate-like crystals, 30 to $70 \mathrm{~nm}$ in diameter, and irregularly disseminated areas of soft organic material.

Conclusions: Crystals forming mineral blocks of mostly compact matter are nucleated on organic substrates with their growth controlled by the diffusion of building units through the virtually stagnant layer of interstitial fluid wetting the deposit surface. Preferentially formed precursors of hydroxyapatite, dicalcium, octacalcium and/or amorphous calcium phosphate, are in later stages of deposit development transformed into biological hydroxyapatite.
\end{abstract}

Keywords: Aortic valve; Calcified deposits; Biological hydroxyapatite

\section{Introduction}

Calcific deposits in human natural heart valves have been investigated by various analytical methods, including X-ray diffraction, Fourier transform infrared and Raman spectroscopy, energy dispersive X-ray, scanning, chemical analysis and highresolution transmission electron microscopy. These methods has shown that the calcifications are several millimetres in diameter with uneven compact surface and highly inhomogeneous interiors without no indication of any morphological order. The deposit interior consists primarily of blocks of macroscopically structureless compact phosphatic phase, macroscopic particles of various sizes and shapes scattered within the porous interior and variable amounts of organic matter. Aortic deposits are composed of both poorly crystalline and amorphous hydroxyapatite (HAP), often carbonated, and occasionally mixed with dicalcium phosphate dihydrate and/or octacalcium phosphate. These deposits are thought to develop by the hydrolysis of precursor phases to biological HAP [1-5].

The crystallinity and chemical and phase composition of the aortic valve calcification studied in this paper have been described previously [6]. This calcification had a high $\mathrm{Ca} / \mathrm{P}$ ratio with non-uniform internal structure composed of large blocks of mostly compact inorganic matter, without distinct internal macroscopic structure, and layers of voluminous organic matter. The inorganic material consisted of amorphous calcium phosphate and poorly crystalline carbonated HAP containing molecular water, with an average formula of $\mathrm{Ca}_{9.1} \mathrm{Mg}_{0.4}(\mathrm{Na}, \mathrm{K})$ $\left(\mathrm{PO}_{4}\right)_{5.8}\left(\mathrm{CO}_{3}\right)_{0.3}(\mathrm{OH})_{2}$.

Electron microscopy studies of the structure of aortic valve deposits have not revealed the mechanism of their formation and development, which is still not satisfactorily understood. The object of this study was to assess the mechanism of formation of aortic valve calcific deposits through an examination of their ultra-fine structure on nanometer scale by atomic force microscopy.

\section{Material and Methods}

\section{Scanning electron microscopy (SEM)}

A human aortic valve calcific mineral deposit, approximately $4 \mathrm{~mm}$ in diameter, was selected from our collection (calcified natural heart valves surgically removed) previously studied (6) and cut in half with a surgical knife. One piece was placed in a vacuum chamber of a scanning electron microscope (a Hitachi S 3400N scanning electron microscope) and its cross-section was observed. The study was a retrospective evaluation of previously obtained surgical samples. Each volunteer provided written informed consent for their clinical information to be published and the study was approved by the ethics committee of our institution.

\section{Atomic force microscopy (AFM)}

The second half of the aortic valve mineral calcific deposit was cast in epoxy resin and cured for several hours at $40^{\circ} \mathrm{C}$. The resulting block of epoxy was not hard enough for preparing a thin slice at ambient temperature and was therefore cut at $-60^{\circ} \mathrm{C}$ in a nitrogen atmosphere using cryo-ultramicrotome (Micro Star Technologies, USA) equipped with a diamond knife. Several smooth slices a few micrometers thick were observed by atomic force microscope (Dimension Icon from Bruker USA) in the Peak Force QNM Imagining Mode which provide a

*Corresponding author: Felix Grases, Laboratory of Renal Lithiasis Research University Institute of Health Sciences Research (IUNICS), University of Balearic Islands, Palma of Mallorca, Spain, Tel: 34971173257; E-mail: fgrases@uib.es

Received January 09, 2014; Accepted January 30, 2014; Published February 01, 2014

Citation: Grases F, Sohnel O, Zelenkova M (2014) Ultrafine Structure of Human Aortic Valve Calcific Deposits. J Cytol Histol 5: 214. doi:10.4172/21577099.1000214

Copyright: @ 2014 Grases F, et al. This is an open-access article distributed under the terms of the Creative Commons Attribution License, which permits unrestricted use, distribution, and reproduction in any medium, provided the original author and source are credited. 
Citation: Grases F, Sohnel O, Zelenkova M (2014) Ultrafine Structure of Human Aortic Valve Calcific Deposits. J Cytol Histol 5: 214. doi:10.4172/21577099.1000214

quantitative nanoscale elastic modulus map. The cross-section was first observed under optical microscope and sites for detailed observation by AFM were randomly selected.

Dark red and light yellow colours on morphological images indicate depressions, i.e. sites lying below the plane of the surface, and projections from the surface, respectively. On nanoscale elastic modulus maps showing variations of the surface modulus (DerjaguinMüller-Toporov or DMT modulus) light yellow and dark red colours indicate areas composed of stiff crystalline and soft matter, respectively. On maps of adhesion indicates of the atomic microscope tip to the sample surface, lighter colours indicate adhesion to the flat soft surface, whereas darker colours indicate weaker adhesion to protruding stiff objects. Small white areas appearing on these maps represent artefacts caused by rebound of the probe tip from the surface on encountering large stiff particles.

\section{Results}

Figure 1a demonstrates disarranged internal structure of the studied aortic valve mineral deposit predominantly composed of large blocks of mostly compact matter without distinct internal microscopic structure. However, observations at higher magnification indicated that this seemingly compact and structureless matter contained cavities and holes and consisted of accumulated small particles, Figure 1b. SEM at
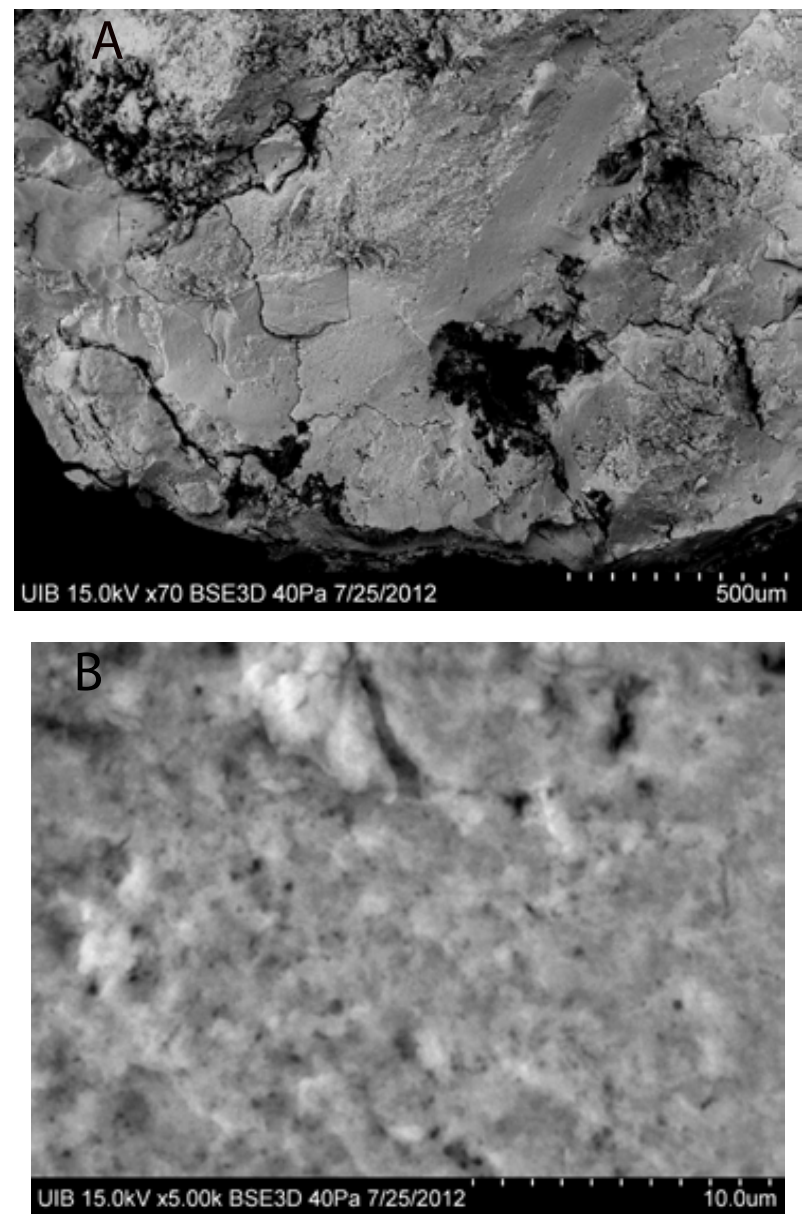

Figure 1: SEM images of the cross-section of an aortic valve calcification. (a) general image of the internal structure.

(b) Detail of the cross-section structure.
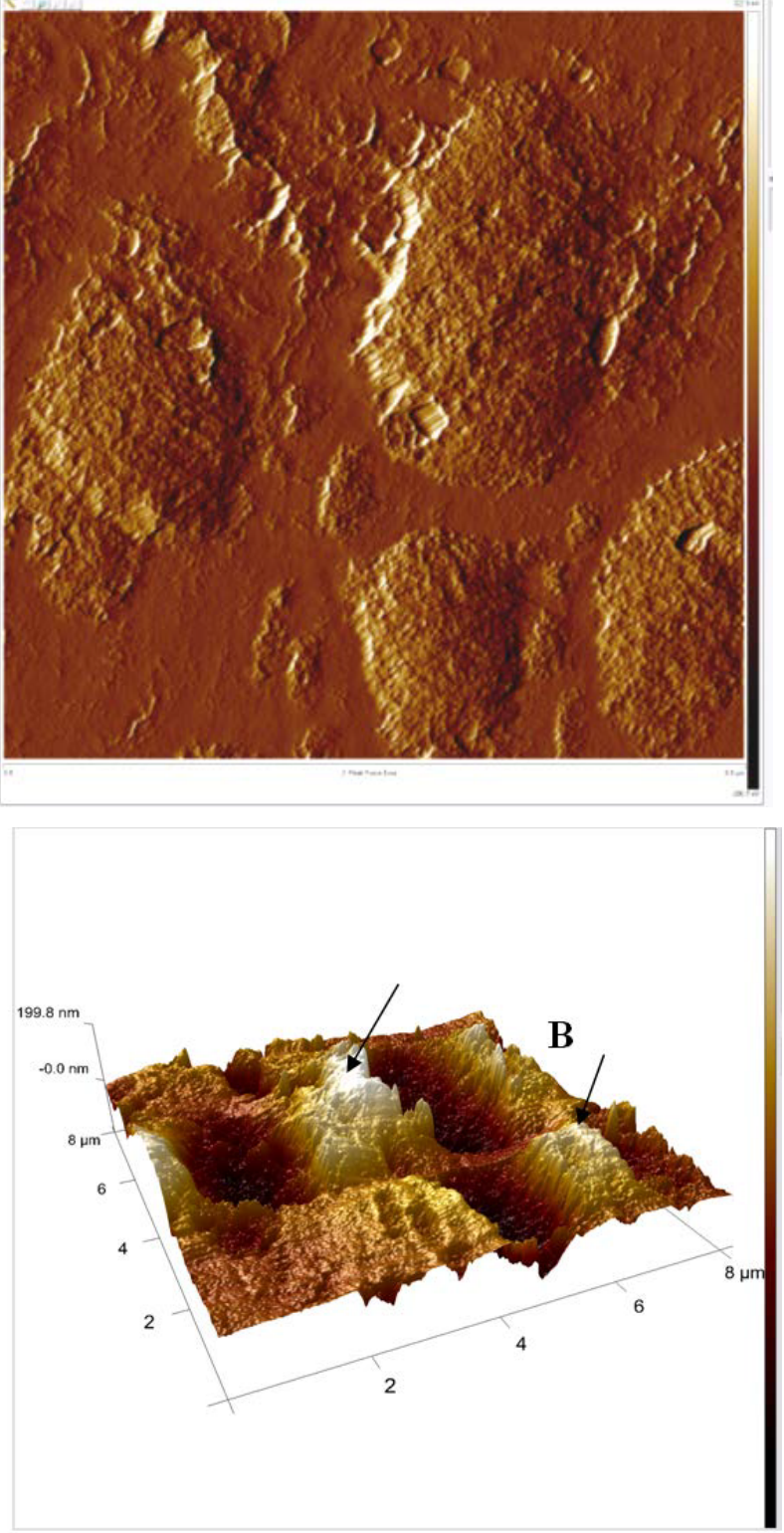

Figure 2: Topographic map of the slice surface, scanned area of $8 \times 8 \mu \mathrm{m}$ (a) Surface visualized in 2-D mode by Peak Force Error showing depressions up to $4 \mu \mathrm{m}$ in size separated by relatively smooth areas (b) 3-D topographic map of the same area.

even higher magnification did not provide further details of the fine structure of these deposits due to unclear and fuzzy images.

A general image of the cross-section surface $8 \times 8 \mu \mathrm{m}$ in size, shows an uneven surface with hills and deep depressions, Figure $2 \mathrm{a}$. Hills with heights up to $200 \mathrm{~nm}$, formed by closely connected columnar crystals, were separated by deep valleys up to $4 \mu \mathrm{m}$ in size, Figure $2 \mathrm{~b}$. The horizontal flat plane, pointed to by arrows in Figure 2b, discernible on the top of the highest columnar crystals, resulted from removal of the crystal tips during slice preparation. Smooth and rough areas were also observed (left and right side lower corners of Figure $2 \mathrm{~b}$.

A more detailed image of the cross-section surface showed that the smooth area was formed by rounded tips of closely packed particles, 
Citation: Grases F, Sohnel O, Zelenkova M (2014) Ultrafine Structure of Human Aortic Valve Calcific Deposits. J Cytol Histol 5: 214. doi:10.4172/21577099.1000214

Page 3 of 5
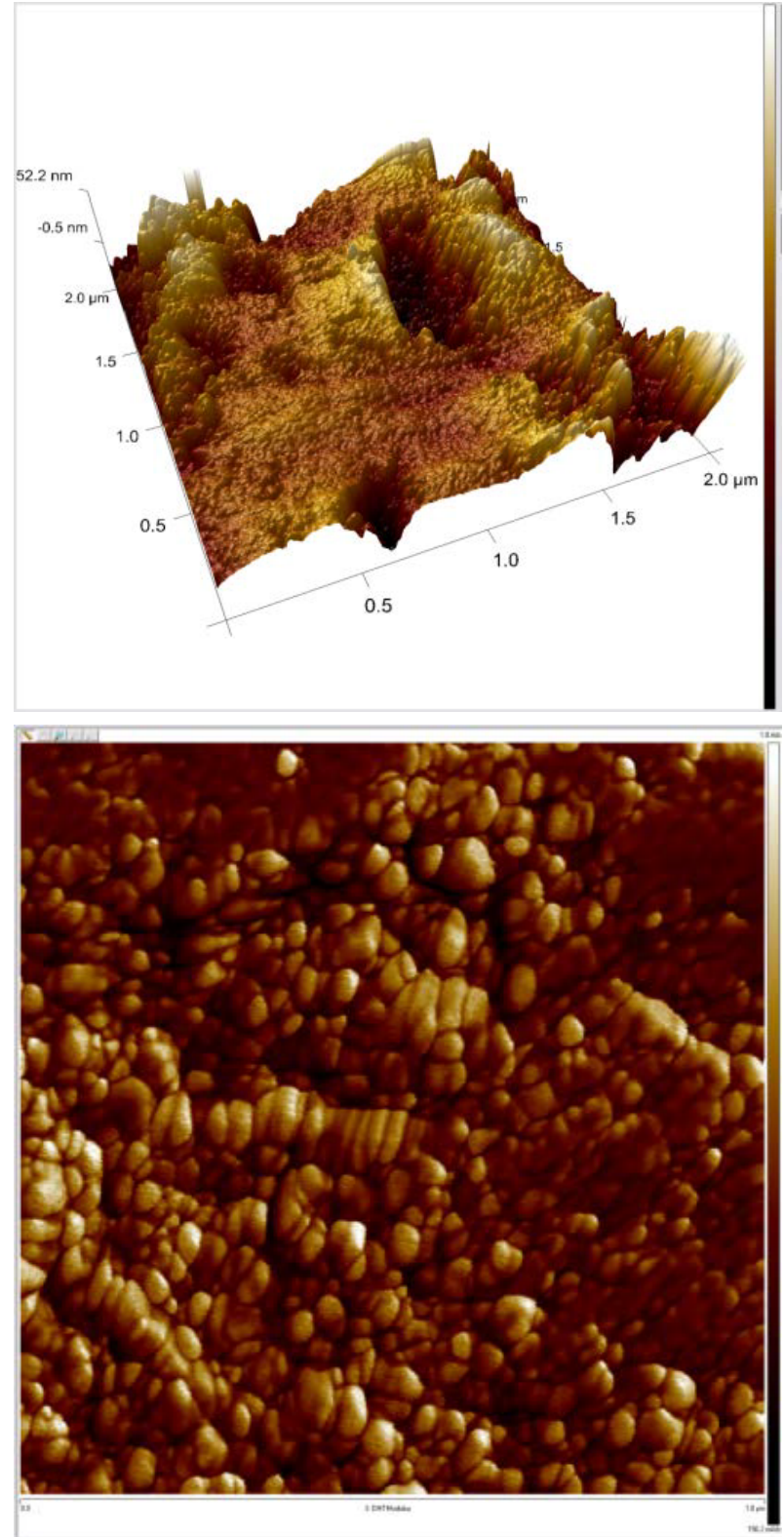

Figure 3: Map of a $2 \times 2 \mu \mathrm{m}$ area of the cross-section composed of rough and smooth surfaces

(a) 3-D topographic map

(b) quantitative nanoscale elastic modulus map of the same area

Figure 3a. These elongated needle-like particles (crystals) had circular or elliptical cross-sections of diameters ranging from approximately 30 to $70 \mathrm{~nm}$, Figure 3b. Blocks of plate-like thin particles (crystals) attached by lateral faces were also observed in Figure $3 \mathrm{~b}$ when viewing the map of the elastic modulus of the same area as in Figure 3a. Soft material (dark red) often enveloped particles consisting of stiff material (light yellow) and also formed larger spots irregularly distributed over the cross-sectional surface.

Details of the smooth and rough areas are shown in Figures $4 \mathrm{a}$ and $4 \mathrm{~b}$. Smooth areas with height differences of less than $6 \mathrm{~nm}$ consisted of small vague objects less than $20 \mathrm{~nm}$ in diameter without clearly defined edges. An adhesion map of the same area, Figure 5 a, confirmed its planarity (light brown colour demonstrates good adhesion), as well as the softness and size of the constituting particles. Rough areas were composed of welldefined closely connected crystals, 30 to $70 \mathrm{~nm}$ in diameter, protruding from the surface. Slight adhesion of the microscope tip to this surface (dark brown colour), Figure5b, corroborates roughness and stiffness of the rough area of this cross-section surface.

\section{Discussion}

The studied slices were cut from the interior of the aortic valve deposit, specifically from a large block of macroscopically compact matter. Since the surfaces of these cross-sections contain flat areas as well as valleys and hills, macroscopically compact matter is in fact porous and contains closed empty cavities. The compact matter is composed of stiff and soft matter. The stiff matter consist of closely adjoining well developed crystalline columnar particles, predominantly needles and some plate-like particles. Soft matter, assumed to be of organic origin, is irregularly distributed throughout the compact matter volume.

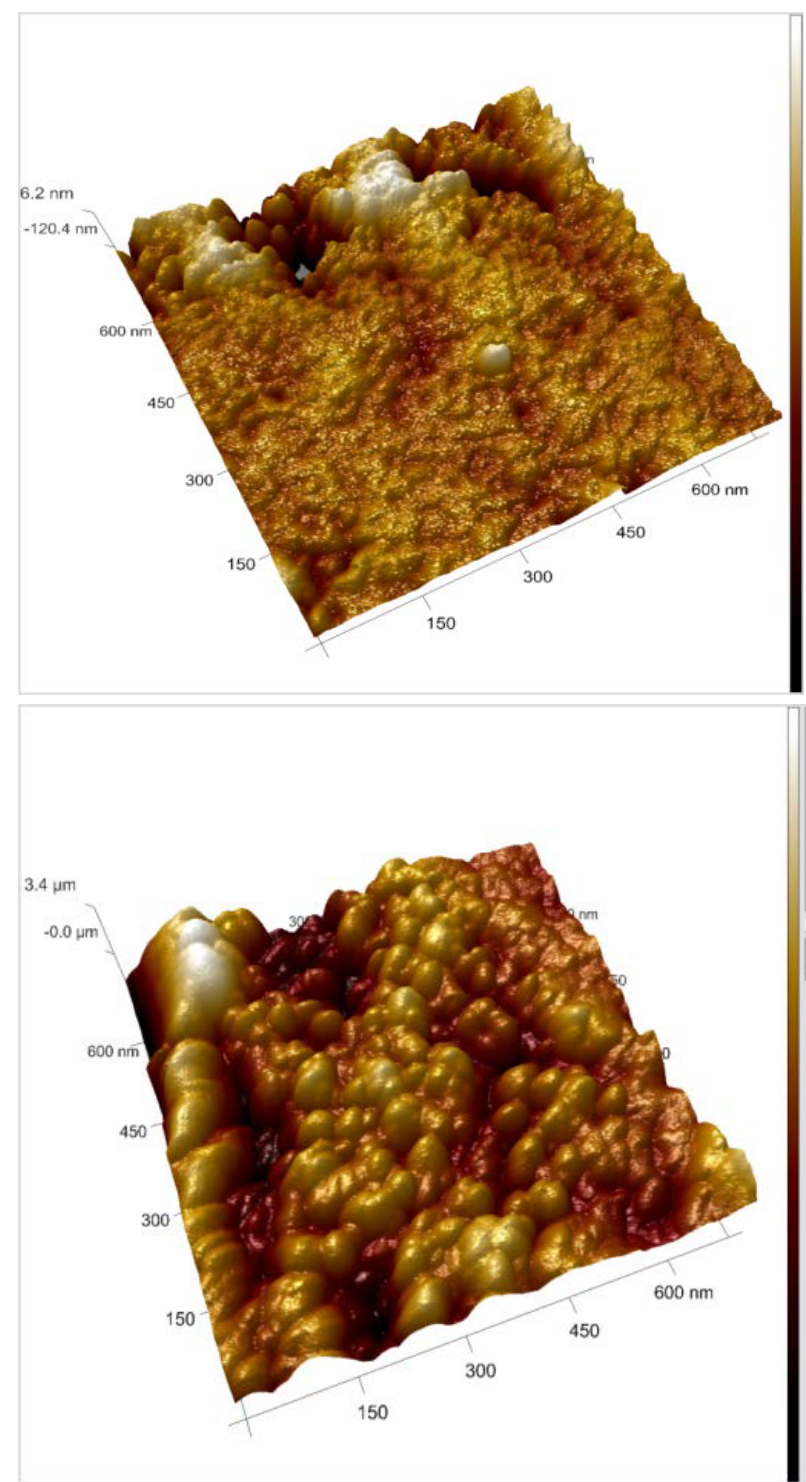

Figure 4: 3-D topographic map of a smooth (a) and a rough (b) surface of the cross-section. Scanned area $0.7 \times 0.7 \mu \mathrm{m}$. 

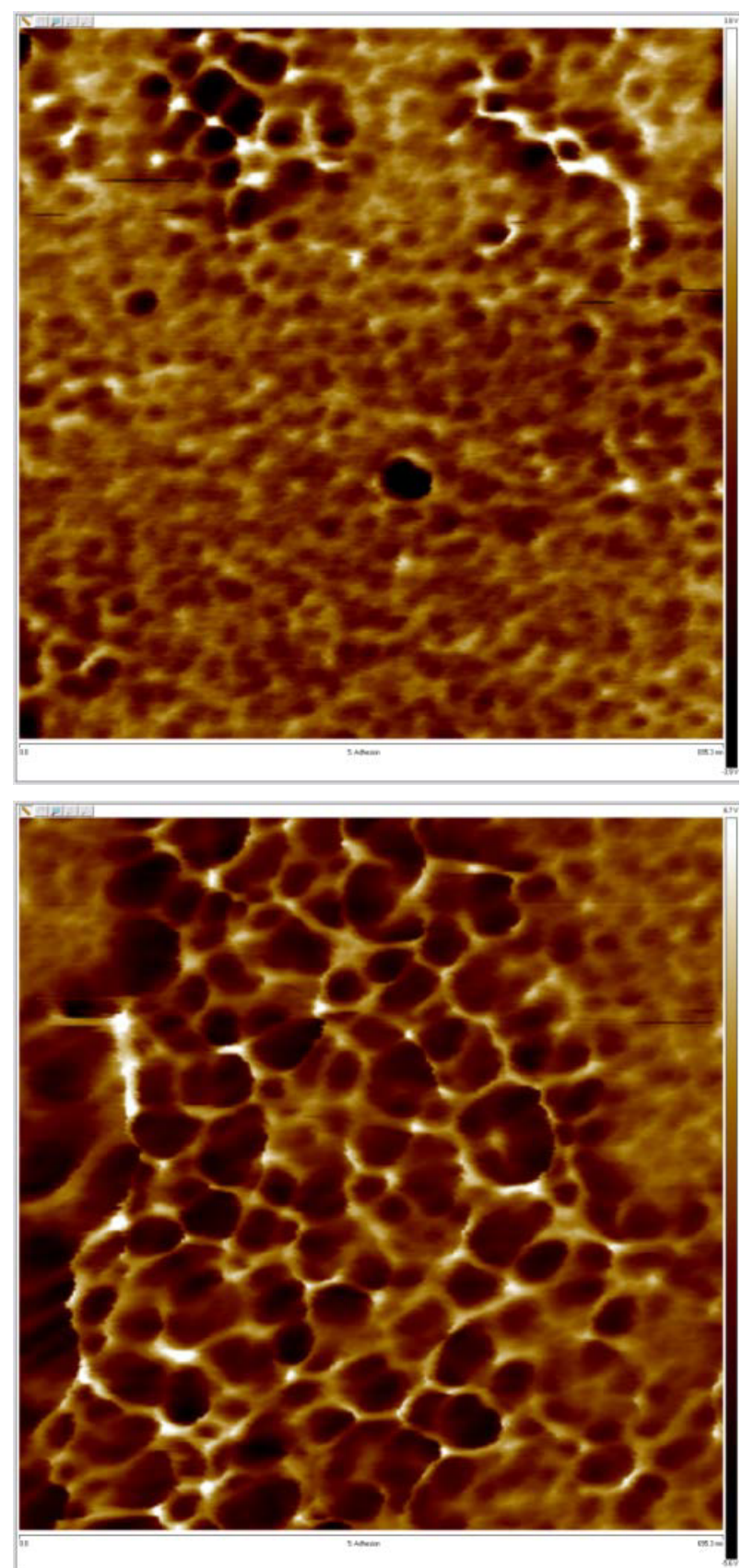

Figure 5: Map of adhesion of (a) smooth and (b) rough surfaces of the crosssection. The observed areas were identical to those in Figure: 3 . Scanned area $0.7 \times 0.7 \mu \mathrm{m}$.

Individual and/or connected spherical particles, formed through aggregation of calcium phosphate clusters in liquid phase by perikinetic coagulation [7], do not occur in aortic valve calcific deposits. This finding suggests that the compact phase occurring in aortic deposits is formed by a mechanism different from that described above.

The first stage of aortic deposit formation from supersaturated interstitial fluid consists of heterogeneous nucleation of solid phase on an organic substrate. Further development proceeds in a very limited volume of interstitial fluid adjacent to the solid phase surface; in fact the surface is just wetted by a thin layer of fluid. Renewal of interstitial fluid in the valve interior by diffusion from the blood stream very slow, making the flow rate over the solid surface is extremely slow. Development of concretion in a virtually stagnant liquid of negligible volume proceeds by transportation of building units of the solid phase to its surface by volume diffusion. Hence, the growth of the solid phase is controlled by volume diffusion, a rather slow process. The slowly growing solid phase can adopt the crystalline habitus typical for a respective compound.

Organic matter, present throughout the interior of the aortic valve concretion, serves as a template for nucleation of crystals [8]. Crystals nucleated on organic matter incorporated into aortic concretions subsequently grow and develop into columnar crystals. The shape of crystals appearing in the compact phase differs from habitus of HAP crystals, suggesting that metastable phases, such as dicalcium phosphate, calcium octaphosphate and amorphous calcium phosphate, are formed preferentially and subsequently are gradually transformed into a thermodynamically stable phase, biological HAP [1,9]. The time available for crystal growth and subsequent transformation is sufficient since aortic concretions develop over several months.

Comparison of the ultra-fine structure and growth conditions of aortic deposits with those of synthetic calcium phosphate concretions [10] and phosphate renal calculi [11], showed two distinctly different mechanisms of phosphate deposit formation from stagnant liquid supersaturated with respect to HAP can be distinguished: (i) Formation in an adequate volume of liquid, such as the formation of phosphate calculi in a kidney cavity with poor urodynamics [11] or the development of synthetic calcium phosphate concretions in simulated body fluid [10].

Phosphate renal calculi are composed predominantly of spherical agglomerates with diameter in the range of 150-300 $\mathrm{nm}$ and large blocks of "structureless" matter with both phases consisting of nanoparticles approximately $10 \mathrm{~nm}$ in diameter. Synthetic calcium phosphate concretions are composed of closely connected round spherical and elliptical objects of diameter varying from 70 to $120 \mathrm{~nm}$ with surface layers composed of tightly packed spherical objects $25-$ $30 \mathrm{~nm}$ in diameter. The predominant mechanism of deposit formation was in both cases settling of larger calcium phosphate aggregates onto and their subsequent incorporation into deposit. These larger aggregates originate by coagulation of ionic cluster, so called Posner's clusters $\mathrm{Ca}_{9}\left(\mathrm{PO}_{4}\right)_{3}$ or $\left[\mathrm{Ca}_{3}\left(\mathrm{PO}_{4}\right)_{2}\right]_{\mathrm{n}}$, which are spontaneously formed in solutions supersaturated with respect to HAP. About $50 \%$ of the HAP in excess of equilibrium is bound into these clusters [12]. Formation of clusters can be enhanced by glycoproteins, such as fetuin [13] and reduced by negatively charged ions, such as citrate and phytate $[14,15]$. This mechanism requires an adequate volume of liquid in contact with a concretion and the absence of liquid movement that would substantially disturbs settling of agglomerates. In heart valves, when in presence of little cavities, such structures (manifested by the presence of spherulites) can be developed [6].

(ii) Formation in a strictly limited volume of liquid; this mechanism occurs in aortic valve deposits formation which are composed of crystalline matter without occurrence of nanometer sized round objects, as the case reported in the present study. In fact the mineral structures found in the present paper were similar to the mineral deposits observed by other authors [16].

Calcium phosphate clusters formed in blood supersaturated with respect to HAP have a low probability to diffuse through artery walls due to their size that is significantly larger than that of phosphate 
anions. Clusters remaining in the blood stream from are probably eliminated by the liver. Formation of these calcium phosphate clusters in blood reduces the concentrations of phosphate and calcium free ions decreasing the supersaturation of fluid which diffuses into the valve interior and is in contact with the deposit. Further formation of clusters in a negligible volume of slightly supersaturated plasma is virtually stopped. The growth of these deposits proceeds by incorporation of free calcium and phosphate ions delivered to the surface by volume diffusion. Under these conditions crystalline phase with a nearly equilibrium habitus originates, as observed in human aortic valve deposits.

\section{Acknowledgements}

This study was supported by a grant from University of Balearic Islands and by the project CTQ2010-18271/PPQ from the Ministerio de Ciencia e Innovación (Gobierno de España), FEDER funds (European Union) and the project gran 9/2011 from Conselleria d'Educació, Cultura i Universitat (Govern de les Illes Balears).

\section{References}

1. Tomazic BB (2001) Physiochemical principles of cardiovascular calcification. Z Kardiol 90: 68-80.

2. Mikroulis D, Mavrilas D, Kapolos J, Koutsoukos PG, Lolas C (2002) Physicochemical and microscopical study of calcific deposits from natural and bioprosthetic heart valves. Comparison and implications for mineralization mechanism. J Mater Sci Mater Med 13: 885-889.

3. Delogne C, Lawford PV, Habesch SM, Carolan VA (2007) Characterization of the calcification of cardiac valve bioprostheses by environmental scanning electron microscopy and vibrational spectroscopy. J Microsc 228: 62-77.

4. Gilinskaya LG, Okuneva GN, Vlasov YuA (2003) Investigation of pathogenic mineralization on human heart valves. II. ESR spectroscopy. J Structural Chem 44: $813-820$
5. Gilinskaya LG, Rudina NA, Okuneva GN, Vlasov YuA (2003) Pathogenic mineralization on human heart valves. III. Electron microscopy. J Structura Chem 44: 1038-1045.

6. Prieto RM, Gomila I, Söhnel O, Costa-Bauzá A, Bonnin O, et al. (2011) Study on the structure and composition of aortic valve calcific deposits: Etiological aspects. J Biophys Chem 2: 19-25.

7. Söhnel O, Garside J (1999) Precipitation: Basic principles and industrial applications. Butterworth, Oxford, UK

8. Giachelli CM (1999) Ectopic calcification: gathering hard facts about soft tissue mineralization. Am J Pathol 154: 671-675.

9. Lu X, Leng $Y(2005)$ Theoretical analysis of calcium phosphate precipitation in simulated body fluid. Biomaterials 26: 1097-1108.

10. Grases F, Zelenková M, Söhnel O (2014) Structure and formation mechanism of calcium phosphate concretions formed in simulated body fluid. Urolithiasis 42: 9-16.

11. Zelenková M, Sohnel O, Grases F (2012) Ultrafine structure of the hydroxyapatite amorphous phase in noninfectious phosphate renal calculi. Urology 79: 968.

12. Oyane A, Onua K, Kokbo T, Ito A (1999) Clustering of calcium phosphate in the system CaCl2-H3PO4-KCl-H2O. J Phys Chem B 15: 6557-6562.

13. Wu ChY, Martel J, Young D, Young JD (2009) Fetuin-A/Albumin-minera Coplexes resembling serum calcium granules and putative nanobacteria: Demonstration of a dual inhibition-seeding concept. Plos One 4: 1-40.

14. Shima F, Uto T, Akagi T, Baba M, Akashi M (2013) Size effect of amphiphilic poly( $\mathrm{\gamma}$-glutamic acid) nanoparticles on cellular uptake and maturation of dendritic cells in vivo. Acta Biomater 9: 8894-8901.

15. Genesan K, Epple M (2008) Calcium phosphate nanoparticles as nuclei for the preparation of colloidal calcium phytate. New J Chem 32: 1326-1330.

16. Jastrzebska M, Zalewska-Rejdak J, Mróz I, Barwinski B, Wrzalik R, et al. (2006) Atomic force microscopy and FT-IR spectroscopy investigations of human heart valves. Gen Physiol Biophys 25: 231-244. 